\title{
Study on Implication of Product Form on Users' Psychology
}

\author{
Youqiang $\mathrm{Xu}$ \\ South China University of Technology \\ Guangzhou, China 510006
}

\author{
Huabin Wang \\ South China University of Technology \\ Guangzhou, China 510006
}

\begin{abstract}
With the continuous development of science and technology, the market products are dazzling. The user's demand for product spirit level is constantly improving. Whether the product form can meet the aesthetic needs of consumers is worth exploring under the market background. In addition to the purpose of expressing its functionality, the product form conveys the cultural connotation of the product through its semantic features, reflecting the aesthetic trend of the society and the value orientation of the user. The product form includes the visual shape, color, materials, etc. of the products, all of which have different degrees of influence on the user's psychology, giving the user different psychological hints and aesthetic intentions. In the process of product development and design, the designer should balance the psychological characteristics of different consumer groups to make the products that users love and have market competitiveness.
\end{abstract}

Keywords—product form; semantics; user's psychology

\section{INTRODUCTION}

Since the industrial revolution, with the advancement of science and technology, the design, function, structure and other aspects of various products have been gradually improved and developed. The product form includes the shape, color and material of the product. The user's requirements for the product are not only reflected in the use and the basic form of the product. The psychological feeling brought by the product form is gradually paid attention to by the user. In addition to the use requirements, it will also have an impact on the user's psychology and even the entire environment. The different forms of the same product will also have different feelings on the user's physiology and psychology, giving users different psychological cues and aesthetic intentions. Therefore, rational product model design on the basis of comprehensive human-machine-environment can not only satisfy the user's demand for basic functions of the product, but also positively suggest the user's psychology and meet the physiological and psychological needs of the user. Studying the impact of product morphology on user's psychology is extremely important for product development and iteration in the future.

\section{Morphological Design In PRODUCt SEMANTICS}

\section{A. Overview of Product Form}

As the key to the transmission of product information, product form can raise the inherent quality of the product, the connotation of organizational structure and other essential factors into external appearance factors, and create the physiological and psychological process through vision [1]. Visual styling, function, structure, craft materials and color are the most important components of the product form. Visual modeling is the most intuitive visual experience of the product for the users. The function is the core part of the product, and the essential attribute of a product. Different functions create a variety of products. The structure is the internal framework of a product. Just like the human body, there are skeletons inside, meridians and many other elements intertwined to form a complete internal structure to ensure the smooth operation of the entire system. The materials and processes, as well as the shapes are related to the user's visual perception of the entire product. First of all, material is the basis of the process. For the same material, the application of different technical processes will make the whole product rich in layering. Secondly, using the same technology, different materials will make the whole product form have great differences. It is an important tool to enhance the sense of product hierarchy and an important factor affecting the user's psychological feelings.

\section{B. Morphological Design in Product Semantics}

Product semantics recognizes and studies industrial products based on the cognitive concept of products. Its design symbolic language is mainly embodied in the form [2]. And the form is exactly what a product should pay attention to. Thus, it can connect with the participating users. The most fundamental form of product form semantics is to express a product clearly through the design of visual features such as color and materials. That is its function, operation, and the usage of the environment. At the same time, more and better cultural connotation should be conveyed to users through more clear and intuitive design method.

For a certain product, the role of product form semantics is mainly reflected in the following aspects: communication between product function information and user, communication between product operation information and 
user, and the communication between product culture information and user. Taking the product "bamboo cup" as an example, its shape is the same as ordinary water cup. However, the function indication is very strong. Cup mouth evolved from the bamboo joint, and the product operation information is clear. In addition, the material is changed to bamboo. The whole product is bamboo blue. There is a kind of natural fragrance, adding cultural heritage to the whole product.

\section{PRODUCT Form DESIGN AND USER'S PSYCHOLOGY}

After a product meets the basic needs of users, it should shift its focus from the physical level to the spiritual level, and pay more attention to the user's psychological level. The product ultimately serves people and should bring the soul interaction to the user. The products are not a bunch of cold tools. Designers will first give the user visual impression based on their own understanding of the aesthetics of the product and the current trend of the public aesthetics, such as the product's "size", color matching, materials, etc. The whole form will also have some influences on user's psychology. It is cool, practical, oppressive, and energetic, dull and so on. When the user purchases the product, the user begins to interact with the product. In this process, it involves the comfort and difficulty of the product operation. It directly affects the user's experience of the product, also has different degrees of influences on user's psychology. In addition, the cultural heritage and connotation displayed in the product form design also need to be conveyed to the users. For the users, these can also play certain positive psychological suggestion.

\section{A. Color Hints on User's Psychology}

After the product has the corresponding visual shape, the use of different colors and their combinations will have positive or negative impact on the user's psychology to a certain extent. The appropriate color combination will make the user feel happy. At the same time, the color can express the temperament of the whole product and the layering of the product. It determines the user's preference for the product. Secondly, when using relevant colors in products, the designers should consider the users in different ages, different environments, and even different countries and ethnic groups. Users will have different psychological feelings for different colors, and different lightness and saturation will produce different psychological feelings. The color has no love, good and evil, true or false, good or bad. People have this kind of psychological effect. People often anthropomorphize colors to express certain meanings. These colors become the symbol of certain things. Over time, people will have kind of prejudice [3]. In general, there are following situations:

Warm colors, including red, orange and yellow, will give the user a warm and healthy feeling. In general, the more yellow components in the color are, the stronger the users will have warm feeling.

Cool colors, including blue, purple, black, etc., will give users a sense of fear, cold and other psychological feelings to a certain extent. In addition, excessive use of single black can cause frustration, and strengthen negative emotions and thoughts. The black should be mixed with other colors.

Moreover, brightness and saturation also affect the user's psychology. Warm colors with too high brightness and saturation can cause discomfort to the user. Conversely, suitable brightness and saturation can also make the user feel happy. These need to be matched and adjusted according to the specific product use environment, the functional properties of the product, and the external environment.

\section{B. Material Implications for User's Psychology}

Materials are the basis of design and the necessary tools for expressing product attributes. In recent years, new materials with superior performance and superiority to traditional materials have been developed or are being studied, such as carbon fiber, nanomaterials, superconducting materials and graphene. The material originally has no emotion, and its emotion comes from people's feelings about its material, that is, texture [4]. Appropriate use can not only express the basic functions of new products, but also have other effects on user's psychology.

Materials can be broadly classified into metals, plastics, wood, glass and cloth. Generally speaking, the surface of the metal-based material is smooth and has no irregularities. And the surface of the metal material will be specularly reflected, exhibiting the metallic texture of the product. The user will have psychological hint of smoothness, coldness, firmness, vitality and scientific sense. In addition, for some metals whose surface has been treated with matte process, the emotion brought by it has some soft changes. At the same time, it makes the product more charming. Plastics are substitutes for natural materials. They are classified into soft plastics and rigid plastics. Soft plastics are thermoplastics. They are hardened by heat and softening and can be repeatedly processed. Hard plastics are thermosetting materials, which cannot be used for secondary processing after heating. The plastics are widely used in daily life. We can see plastics in many products that users touch, including mice, keyboards, printers, rice cookers, etc. Generally speaking, compared with metal users, plastics tend to have a calming atmosphere. Transparent plastics can have beautiful, warm, light and comfortable psychological feeling. Adding different colors, users will have more wonderful mentality. Wood-based materials are more common in interior decoration. They are a variety of products processed from different tree species in nature, giving people a rustic, precious, heavy and expensive feeling. In addition, if the designers preserve the texture, pattern and color of natural wood, the user will have a psychological hint that is closer to nature and more environmentally friendly. Unlike other materials, glass materials are very different. It can reflect light. The glass will allow users to have cold, clean, bright, wonderful psychological hints. Also, the users can feel fragile, sharp psychological feeling. The glass is commonly used in interior and exterior decoration of buildings and other electronic products. Cloth materials are also widely used, such as clothing products, plush toys, etc. Generally 
speaking, users can feel soft, delicate and warm psychological hints.

\section{The Implication of Visual Modeling on User's Psychology}

Visual modeling is one of the most important factors for product success. The trend of the lines and the reasonable proportion are the factors that influence the product form. Based on the requirements of product functions, social aesthetic needs and user's psychology, the designer will make the innovation of product styling. When a user sees a specific product, the user can understand the content expressed by the product and the psychological hints brought by the product. When a user purchases a product, he or she will make a purchase decision based on a certain kind of information expressed by the visual shape to compare with what he or she wants.

First of all, visual styling should conform to the aesthetic needs of the current society, allowing users to pursue simpler and more efficient lifestyle. It will achieve the psychological resonance of users. For example, iphone mobile phone has created a new era of mobile phones, which leads the trend of smart phones worldwide. It has changed the way people use their mobile phones. Mobile phones can really play their huge role. They are not just are communication tools.

Secondly, after the product meets the basic needs of the user, its visual shape should have certain personalization. When the functions of a certain type of product are basically the same, the shape must have personalized element. It can be distinguished from other similar products. It is also necessary to satisfy the individualized psychology of some people. For example, the two intake "nostrils" on the front of a BMW car have become one of the most important signs of BMW cars. This is the most different point for BMW cars to distinguish them from other cars. It is a unique personal element of BMW cars.

\section{ANALYSIS OF APPLICATION EXAMPLES OF DIFFERENT PRODUCT FORMS AND USER'S PSYCHOLOGY}

In daily design, the product form is designed to meet the user's needs through visual modeling, color, materials, etc. Taking into account the user's psychological factors, it will inevitably affect the product form design. The following is an example to illustrate the relationship between different product forms and user's psychology.

\section{A. Different Color Matching and User's Psychology of Product Form}

For a specific product, color will increase the beauty of the product, attract the attention of the user, and also affect the sales of the product and the user's experience. Usually, the designer will design different color combinations to meet the demand of market. At the same time, color matching is also an important carrier of product temperament attributes.

As shown in the following figures, the only thing that changes the Bluetooth sports headphone products from left to right is the color matching. The psychological feelings they bring to the users are different. In "Fig. 1", Bluetooth headset with red and black color matching gives users warm psychological hint. It is more suitable for female users. In "Fig. 2", Bluetooth headset with green and black color matching gives users a sense of vitality and sports. It is suitable for young people who like to show their personality. In "Fig. 3", Bluetooth headset with blue and black color matching gives users a more "scientific sense of technology". In "Fig. 4", Bluetooth headset with whole black gives the users weak psychological suggestion of vitality and health, and obvious sense of stability.

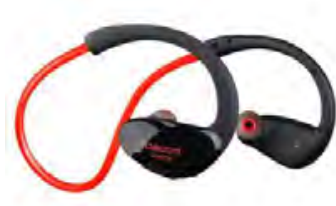

Fig. 1. Bluetooth sports headphone.

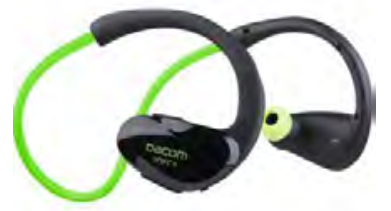

Fig. 2. Bluetooth sports headphone

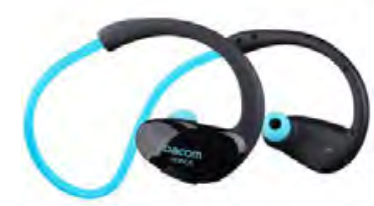

Fig. 3. Bluetooth sports headphone.

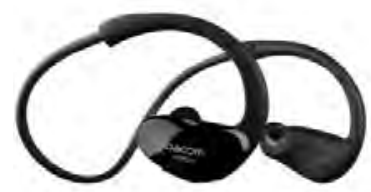

Fig. 4. Bluetooth sports headphone

a. picture source : https://item.jd.com/1510055030.html?jd pop=f3bb7146-4f7b-4d8f-98dd$78 \mathrm{e} 15196 \mathrm{f} 4 \mathrm{a} 5 \& \mathrm{abt}=0$

In product design, the design of human-computer interaction interface is consistent with user's habits, making color an important tool for distinguishing product function operations. When users face a new product, they often see the new human-machine interface and don't know how to 
start the operation, as shown in "Fig. 5". By comparing the color buttons on the operation panel, the user can easily know that it is a switch button. Its color effect is the strongest. And it is in the most important position for the user's mind. "Fig. 6" shows the subway ticketing instruction interface. Different functions are distinguished by different colors. Therefore, even if the user is the first time to make the operation, the ticket can be purchased according to the color button on the interface. "Fig. 7" is the treadmill operation interface. The opening button is designed to be green, and the stop button is designed to be red. In addition to the happiness, red will make people have nervous and dangerous psychological hints. Green is more natural and safe, as is the traffic lights on the street.
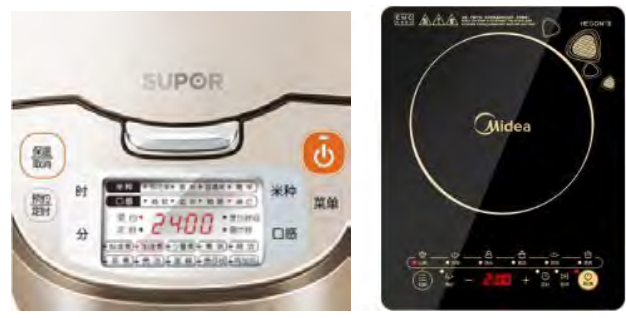

Fig. 5. The operation pane of human-machine interface.

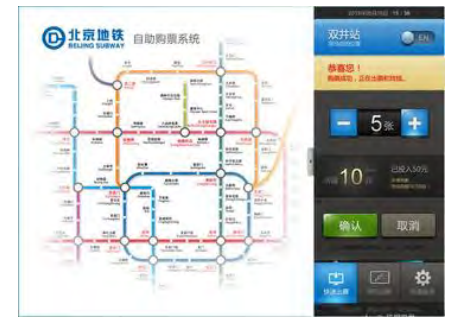

Fig. 6. The subway ticketing instruction.

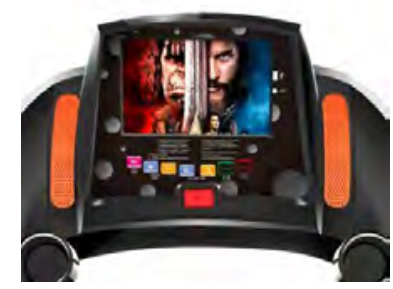

Fig. 7. The treadmill operation.

picture source : Baidu picture and pictures from the website https://www.jd.com/

\section{B. Different Materials and User's Psychology of Product Form}

There are many kinds of products on the market, and different materials can reflect different layers of products. In addition to their different functions, the texture of the products displayed by the materials is not the same. And the psychological hints that users get from them are different.

For example, the psychological suggestion of glass and marble is different, as shown in the following figures. "Fig. 8 " is a glass-based building, giving people a cool visual experience, and people will have light, novel, clean, and bright psychological hint. It is very consistent with the social background of fast-paced life. To a certain extent, it can alleviate the work pressure of young people in modern society. In contrast, the building in "Fig. 9" uses marble as the main material, which is full of heavy feeling and gives people a solid and stable psychological feeling. In addition, the psychological implication of using metallic materials in products is not the same as the psychological suggestion of using plastics. "Fig. 10" and "Fig. 11" show glass water cups. In "Fig. 10", it uses a metal bottle cap to add certain vitality to the whole product. At the same time, it gives the user a strong and cold feeling. In "Fig. 11", it adopts the black plastic bottle cap, which will be more psychologically calmer for the user. For this product, the user will even feel the relative oppression. The overall visual effect will be weaker on the left side, and the products on the left will be more favored by users. Its sales volume will naturally be better, which needs to be considered before the product is put on the market.

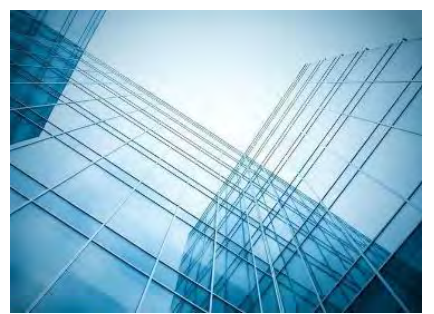

Fig. 8. A glass-based building.

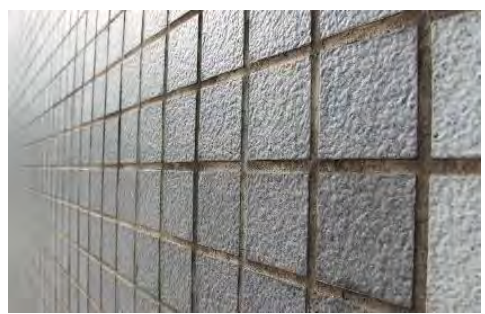

Fig. 9. A marble-based building.

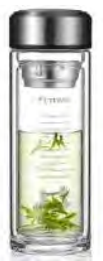

Fig. 10. Glass water cups.

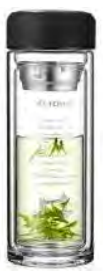

Fig. 11. Glass water cups. 


\section{Different Visual Shapes and User's Psychology of Product Form}

After the designer gives the basic function of a certain product, the process of giving the color of the material is followed; the overall visual shape is still a key factor affecting the user's experience.

Since the development of bicycles from the last century to the present, the product form has been greatly improved. The visual modeling, material processing, color matching, etc. of bicycles have become more and more various. There are also many kinds of bicycles, such as mountain bikes, road bikes, leisure bicycles, fixed cog, folding bicycles, etc. They greatly satisfy the individual needs of users. From the perspective of visual styling, it is mostly based on simple style, which is very sporty and more in line with the fastpaced lifestyle of modern society. At the same time, there are different combinations of colors, instead of being monotonous as before. It fully meets the basic needs of users. In addition, users can even order a bicycle that suits their personality. We can take Phoenix bicycle as an example. In "Fig. 13", there is a newer Phoenix mountain bike. It uses blue, yellow and black colors to make the whole bicycle livelier. The shape gives the user a cool, energetic and unassuming feeling. In addition, many parts of this type of bicycle have been simplified, which is compared to the past. And this kind of bicycles gives users a streamlined psychological suggestion. The material is mainly made of aluminum alloy, which will not make the whole bicycle thick and heavy. It gives users the suggestion of lightness and fast tips. In "Fig. 14", it is a road bike. The red, yellow and black color matching is very bright and eye-catching. When designing such a bicycle shape, the designer should consider the users of the bicycle and its use. Therefore, this kind of bicycles first give people a sense of vitality, which is in line with the characteristics of the users who pursue speed and passion. Moreover, the handlebar of the bicycle is different from that of other bicycles, giving people psychological suggestion of going forward and quick. In "Fig. 15", it is a folding bicycle, which gives a low-level, casual, safe and light psychological hint from the visual shape. It is not as individual as the previous one. They are relatively simple and basically capture the aesthetic psychology of modern users.

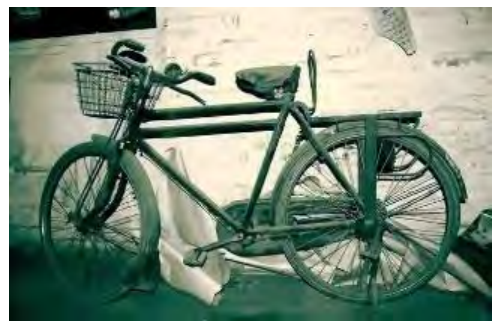

Fig. 12. Old bick.

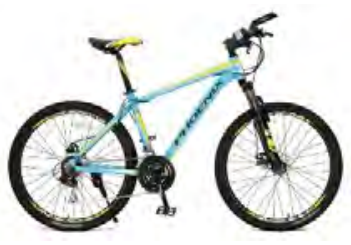

Fig. 13. Phoenix mountain bike.

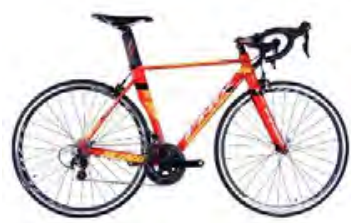

Fig. 14. A road bike.

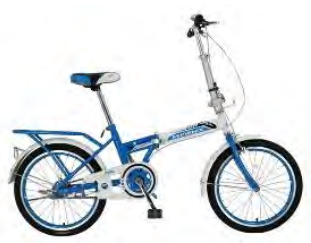

Fig. 15. A folding bicycle

a. picture source: http://www.phoenix-bicycle.com.cn

"Fig. 5" "Fig.9" shows a series of product forms, and they have reflected different user's psychology. In the first quadrant of the figure, the product form is mainly rounded, and the surface of the product changes more diversely. At the same time, it has a fashionable shape, which is more in line with the aesthetics of modern society, meets the user's visual desire, and gives the user a safe and soft psychological hint. The fourth quadrant in the figure is similar to the first quadrant. In terms of product characteristics, it is not in line with the current user's aesthetics. And the overall style of the product will be dull under the soft and round shape. In the second quadrant, the product also has a fashionable shape. From a visual point of view, its product line trend is more sharp, giving users a hard and firm psychological feeling. The third quadrant in the figure has the same surface line as that of the second quadrant, which gives the user a sharp or even dangerous psychological suggestion. 


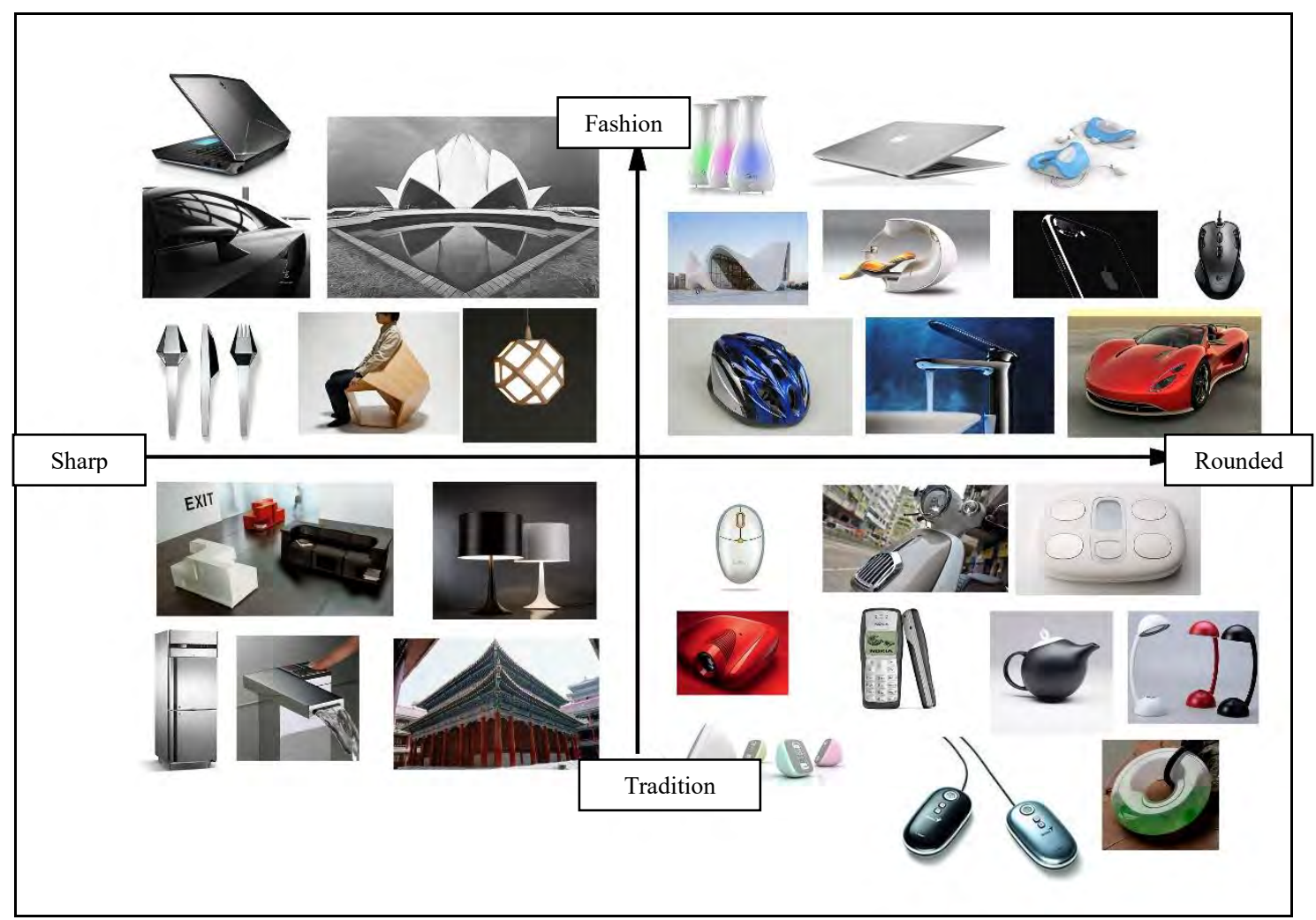

Fig. 16. A series of product forms. (made by the author).

\section{CONCLUSION}

The user is in contact with the product all the time, and the interaction behavior occurs. This process first satisfies the user's use requirements. More importantly, it takes care of the user's psychological needs. The product form is inseparable from the user's psychology. The design and development process is in progress. The impact of research product morphology on user psychology is crucial. With different material techniques, visual shapes and color combinations, the users will have different psychological feelings. In addition, the initial design should fully understand the psychological needs of the user, and make the innovation in terms of structure, function, production, etc. And then, it will meet the different needs of users. The users will understand the nature of the design. Both parties can benefit from it [5].

\section{REFERENCES}

[1] Yin Hang. Analysis and continuation application of product form [D]. Jiangnan University. 2007

[2] Bai Xiaojian. Research on design method based on product morphological semantics [J]. Popular Literature, 2012(05): 56-57.

[3] Zhang Fan.The importance of color in product design[J]. Journal of Shanxi Coal Management Cadre College, 2007(03):146-147.

[4] Chen Hongjuan. Application Research on Material Emotional Characteristics in Product Design [J]. Packaging Engineering, 2011(10): 59-62.
[5] Ning Shaoqiang, Liang Huiping.Exploring on psychology of useroriented in designing products [C]. Ningbo.2004: 226-232

[6] Liu Guoyu, Shen Jie. Basic product form design [M]. Beijing: China Light Industry Press, 2001.

[7] Hu Xiaoqing, Wang Miaohui, Gao Yunfang. Feeling characteristics and product form design of metal materials[J]. Packaging Engineering, 2007,28(8):184-186.

[8] Liu Sha. Design art psychology [M]. Beijing: Tsinghua University Press, 2006.

[9] Song Ming. With the accumulation of famous brands, continue the "Phoenix Legend" [J]. Electric Bicycle. 2010 (09). 